\title{
Combined Human Umbilical Cord-Derived Stem Cell and Hyperbaric Oxygen Therapy Effectively Preserved the Neurological Function and Brain Architecture in Rat After Acute Intracerebral Hemorrhage
}

Hon-Kan Yip

Chang Gung Memorial Hospital Kaohsiung Branch

Kun-Chen Lin

Chang Gung Memorial Hospital Kaohsiung Branch

Pei-Hsun Sung

Chang Gung Memorial Hospital Kaohsiung Branch

John Y. Chiang

Sun Yat-Sen University

Tsung-Cheng Yin

Chang Gung Memorial Hospital Kaohsiung Branch

Re-Wen Wu

Chang Gung Memorial Hospital Kaohsiung Branch

Kuan-Hung Chen ( $\square$ amigofx35@gmail.com )

Chang Gung Memorial Hospital Kaohsiung Branch

\section{Research}

Keywords: intracerebral hemorrhage, mesenchymal stem cells, hyperbaric oxygen, neurological function

Posted Date: September 3rd, 2020

DOI: https://doi.org/10.21203/rs.3.rs-70183/v1

License: (1) (i) This work is licensed under a Creative Commons Attribution 4.0 International License.

Read Full License 


\section{Abstract}

Background: This study tested the hypothesis that combined therapy with human umbilical cord-derived mesenchymal stem cells (HUCDMSCs) and hyperbaric oxygen (HBO) was superior to either one on preserving neurological function and reducing brain-hemorrhagic volume (BHV) in rat after acute intracerebral hemorrhage $(\mathrm{ICH})$ induced by intracranial injection of collagenase.

Methods and Results: Adult-male SD rats $(n=30)$ were equally divided into group 1 (sham-operated control), group $2(\mathrm{ICH})$, group $3\left(\mathrm{ICH}+\mathrm{HUCDMSCs} / 1.2 \times 10^{6}\right.$ cells/intravenous injection at $3 \mathrm{~h}$ and days $1 / 2$ after $\mathrm{ICH})$, group $4(\mathrm{ICH}+\mathrm{HBO} /$ at $3 \mathrm{~h}$ and days $1 / 2$ after $\mathrm{ICH})$ and group $5(\mathrm{ICH}+\mathrm{HUCDMSCs}-\mathrm{HBO})$, and euthanized by day 28 after $\mathrm{ICH}$. By day 1 , the neurological function was significantly impaired in groups 2 to 5 than in group $1(p<0.001$ ), but it did not differ among groups 2 to 5 . By days $7 / 4 / 28$, the integrity of neurological function was highest in group 1, lowest in group 2 and significantly progressively improved from groups 3 to 5 (all p<0.001). By day 28, the BHV was lowest in group 1, highest in group 2 and significantly lower in group 5 than in groups $3 / 4$ (all $p<0.0001$ ). The protein expressions of inflammation (HMGB1/TLR-2/TLR-4/MyD88/TRAF6/p-NF-KB/IFN- $/$ /L-1ß/TNF-a), oxidative-stress/autophagy (NOX1/NOX-2/oxidized protein/ratio of LC3BII/LC3BI) and apoptosis (cleaved-capspase3/PARP), and cellular expressions of inflammation (CD14+, F4/80+) in brain tissues exhibited an identical pattern, whereas cellular levels of angiogenesis (CD31+/vWF+/small-vessel number) and number of neurons (NeuN+) exhibited an opposite pattern of BHV among the groups (all $\mathrm{p}<0.0001$ ).

Conclusion: combined HUCDMSC-HBO therapy offered better outcomes after rat ICH.

\section{Background}

Intracranial hemorrhage, one kind of stroke resulted from subarachnoid hemorrhage or intracerebral hemorrhage $(\mathrm{ICH})$, is a highly unacceptable cause of death and disability in adults with an annual incidence of 10-40 per 100,000 population [1-3]. In intracranial hemorrhage, the bleeding is usually derived from arterioles or small arteries directly into the brain, forming hematomas that spreads along white matter pathway. The hematoma frequently continues to grow until the pressure surrounding it increases enough to limit its spread or until the hemorrhage decompresses itself by emptying into the ventricular system or into the cerebrospinal fluid $[2,4]$. Additionally, intracranial hemorrhage, always sabotages the brain tissue as it enlarges, i.e., first primary injury to the brain is the mechanical damage [2]. The pressure created enough by blood and surrounding brain edema is life-threatening [4]. The secondary injuries result from cytotoxicity of blood [5, 6], impaired calcium homeostasis [7], excitotoxicity from excitatory neurotransmitters (e.g. glutamate) [8,9] and oxidative stress and inflammation [6, 9-14]. This neuroinflammation is upregulated, resulting in the release of cytokines, chemokines, cellular adhesion molecules (CAMs), and matrix metalloproteases (MMPs) [15]. Expression of MMPs increases the permeability of the BBB, allowing peripheral leukocytes to invade the area of injury, where they upregulate present inflammatory processes [15]. Additionally, CAMs allow leukocytes to adhere to local vessels, permitting those cells to attract more cells to the site of injury [15]. These aforementioned issues 
which are complicated could explain why the effective treatment of intracranial hemorrhage is still unmet need.

Hypoxic neurons performing anaerobic metabolism result in acidosis, lactate production, and an unsustainable reduction in cellular metabolic reserve [16]. As the hypoxic microenvironment persists, the neuronal cells lose their ability to maintain ionic homeostasis, follow by free oxygen radical accumulation and cell membrane degradation [16], resulting in an irreversible cell death [[16]. This gives some basis to the assertion that therapy designed to increase oxygen availability in the early period following brain hemorrhage may improve long-term outcomes [17]. Interestingly, hyperbaric oxygen therapy (HBO) has been reported to be effective in improving the outcomes after traumatic brain injury [17]. Other study has reviewed that HBO could be a new look on treating ischemic stroke and traumatic brain injury [18]. HBO is the therapeutic administration of $100 \%$ oxygen at environmental pressures greater than 1 atmosphere absolute (ATA) in an airtight vessel. In this way, it is possible to deliver a greatly increased partial pressure of oxygen to the tissue, including the ischemic brain tissues.

Mesenchymal stem cell (MSC) has the capacity to attenuate inflammation [19-24] and down-regulate innate and adaptive immunity [19, 21-26] through suppressing immunogenicity [19, 21-26].

Experimental studies have further demonstrated that MSCs therapy markedly preserved neurological function and reduced brain infarct volume in rodent after acute ischemic stroke attack [27, 28]. The results of these previous reports $[19,21-28]$ raise the hypothesis that MSCs therapy may offer unexpected benefit for patients after $\mathrm{ICH}$, especially for those who have large $\mathrm{ICH}$ zone non-candidate for surgical intervention. However, prior to apply MSC therapy for human being in a clinical setting of $\mathrm{ICH}$, a preclinical study had to be first performed by using human being derived MSC (i.e., xenogeneic MSC) to prove not only the safety and efficacy but also the immune privilege of the MSC regardless for what kind of biological species. Furthermore, due to the complexity of the pathophysiologic mechanisms involved in the hemorrhagic stroke, a single therapeutic strategy would be inadequate for disease improvement. Therefore, we performed a combined therapy with human umbilical cord blood-derived stem cell (HUCDMSCs) and HBO for rodent after acute ICH.

\section{Materials And Methods}

\section{Ethics}

All animal experimental procedures were approved by the Institutional Animal Care and Use Committee at Kaohsiung Chang Gung Memorial Hospital (Affidavit of Approval of Animal Use Protocol No. 2018032102) and performed in accordance with the Guide for the Care and Use of Laboratory Animals, 8th edition (NIH publication No. 85 - 23, National Academy Press, Washington, DC, USA, revised 2011). Animals were housed in an Association for Assessment and Accreditation of Laboratory Animal Care International-approved animal facility in our hospital, with controlled temperature and light cycles $\left(24^{\circ} \mathrm{C}\right.$ and 12/12 light/dark cycle). 


\section{Procedure and protocol of animal model of intracerebral hemorrhage (ICH) induced by type IV collagenase proteolytic enzyme}

The procedure and protocol of ICH was based on previous report [29]. In details, the rats were anesthetized by inhalation of $2.0 \%$ isoflurane and then were placed on a warming pad at $37^{\circ} \mathrm{C}$, followed by securing the head and shaving the scalp hair. Under sterile conditions, $1 \mathrm{~cm}$ long midline incision of the scalp was carefully created to expose the perpendicular intersection point of the coronal and sagittal suture (i.e., bregma). The Hamilton syringe $(250 \mu \mathrm{l})$ was then mounted onto the injection pump and stereotaxically guided the needle ( $26 \mathrm{Gauge}$ ) over bregma. The stereotactic manipulator arms were adjusted the position of the needle $1.4 \mathrm{~mm}$ anterior and $3.2 \mathrm{~mm}$ lateral to the right. A small cranial burr hole was then created by using a $1 \mathrm{~mm}$ drill bit. After this procedure, $1.0 \mu \mathrm{l}$ collagenase type IV ( $0.25 \mathrm{IU} /$ $\mu \mathrm{l}$ ) was then carefully injected into corpus/dorsal striatum ( $5 \mathrm{~mm}$ below the skull) by Hamilton syringe 26 $\mathrm{G}$ at a rate of $0.2 \mu \mathrm{l} / \mathrm{min}$. The syringe was removed slowly after the injection is completed and sterile bone wax is used to plug the hole quickly. The skin on the surface of head was then closed by using $4-0$ prolene suture. Finally, the animals were cared for in a portable animal intensive care unit (ThermoCare®) with food and water for 24 hours.

\section{Animal grouping}

Pathogen-free, adult male Sprague-Dawley (SD) rats $(n=30)$ weighing 320-350 g (Charles River Technology, BioLASCO Taiwan Co. Ltd., Taiwan) were used in the present study. The animals were equally categorized into group 1 [sham-operated control (SC), i.e., only incision of the skin over scalp], group 2 [acute ICH induced by directly intracranial injection of collagenase $(1.0 \mu \mathrm{l})$ ], group $3[\mathrm{ICH}+$ HUCDMSCs $\left(1.2 \times 10^{6}\right.$ cells) by intravenous administration at $3 \mathrm{~h}$ and on days 1 and 2 , respectively after $\mathrm{ICH}$ procedure, i.e., total amount was $\left.3.6 \times 10^{6} \mathrm{cells}\right]$, group 4 [ICH + hyperbaric oxygen therapy (HBO) (3 h duration for each time) at $3 \mathrm{~h}$ and at days 1 to 4 after $\mathrm{ICH}$ procedure] and group 5 (ICH + combined HUCDMSCs + HBO). Animals in each group were euthanized by day 28 after $\mathrm{ICH}$ induction and the brain specimen was harvested from each animal for individual study.

\section{Hyperbaric oxygen therapy}

The procedure and protocol of HBO therapy was based on a previous report [30]. Briefly, to induce tissuelevel hyperoxia, SD rats were subjected to HBO administration in an animal tabletop chamber (PiersolDive, model 4934) with the animals exposed to $100 \%$ oxygen at 2.4 atmospheres absolute (ATA) for 90 minutes ( $3 \mathrm{~h}$ /one time) at $3 \mathrm{~h}$ and days 1 to 4 after $\mathrm{ICH}$ induction. 


\section{Corner test for assessment of neurological function prior to and after $\mathrm{ICH}$ induction}

The sensorimotor functional test (corner test) was conducted for each rat of each group (i.e., $\mathrm{n}=6$ per group) at baseline and on days 1, 7, 14 and 28 after acute ICH induction as we previously described [28, $30,31]$. In detail, the rat could walk through a tunnel and then turn into a 60-degree corner. To exit the corner, the rat could turn either left or right. The results were recorded by a technician blinded to the study design. This test was repeated 10 to 15 times with at least 30 seconds between each trial. We recorded the number of right and left turns from 10 successful trials for each animal and used the results for statistical analysis.

\section{Measurement of brain hemorrhagic area}

To evaluate the impact of HUCDMSCs-HBO treatment on preserving the brain parenchyma, coronal sections of the brain were obtained from four extra animals in each group as $2 \mathrm{~mm}$ slices by day 14 after $\mathrm{ICH}$ induction. Each cross section of brain tissue was then stained with 2\% 3,5-Triphenyl-2H-Tetrazolium Chloride (TTC) (Alfa Aesar) for BIA analysis. Briefly, all brain sections were placed on a tray with a scaled vertical bar to which a digital camera was attached. The sections were photographed from directly above at a fixed height. The images obtained were then be analyzed using Image Tool 3 (IT3) image analysis software (University of Texas, Health Science Center, San Antonio, UTHSCSA; Image Tool for Windows, Version 3.0, USA).

The hemorrhagic area was observed as either whitish or pale reddish regions (i.e., dis-colored region). Intracerebral hemorrhagic region was further confirmed by microscopic examination. The percentages of hemorrhagic area were then obtained by dividing the area with total cross-sectional area of the brain. The rest of the brain tissue was then cut into pieces for specific studies.

\section{Western blot analysis}

The procedure and protocol for Western blot analysis were based on our recent reports [28, 30-33]. Briefly, equal amounts $(50 \mu \mathrm{g})$ of protein extracts were loaded and separated by SDS-PAGE using acrylamide gradients. After electrophoresis, the separated proteins were transferred electrophoretically to a polyvinylidene difluoride membrane (GE, UK). Nonspecific sites were blocked by incubation of the membrane in blocking buffer [ $5 \%$ nonfat dry milk in T-TBS (TBS containing $0.05 \%$ Tween 20)] overnight. The membranes were incubated with the indicated primary antibodies [Caspase 3 (1: 1000, Cell Signaling), Poly (ADP-ribose) polymerase (PARP) (1: 1000, Cell Signaling), high mobility group box 1 (HMGB1) (1: 1000, Cell Signaling), toll-like receptor (TLR)-2 (1: 1000, Abcam), TLR-4 (1: 1000, Abcam), myeloid differentiation primary response 88 (MyD88) (1: 1000, Abcam), TNF receptor associated factor 6 (TRAF6) (1: 1000, Abcam), IKB-ß (1: 1000, Abcam), nuclear factor (NF)-KB (1: 600, Abcam), interferon (INF)- $y$ (1: 1000, Abcam), LC3B-I (1: 1000, Cell Signaling), LC3B-II (1: 1000, Cell Signaling), oxidized 
protein (1:200, Millipore), tumor necrosis factor (TNF)- $a$ (1: 1000, Cell Signaling), interleukin (IL)-1ß (1: 1000, Cell Signaling), NOX-1 (1: 1500, Sigma), NOX-2 (1: 750, Sigma), and Actin (1: 1000, Millipore)] for 1 hour at room temperature. Horseradish peroxidase-conjugated anti-rabbit immunoglobulin IgG (1:2000, Cell Signaling, Danvers, MA, USA) was used as a secondary antibody for one-hour incubation at room temperature. The washing procedure was repeated eight times within one hour. Immunoreactive bands were visualized by enhanced chemiluminescence (ECL; Amersham Biosciences, Amersham, UK) and exposed to Biomax L film (Kodak, Rochester, NY, USA). For the purpose of quantification, ECL signals were digitized using Labwork software (UVP, Waltham, MA, USA).

\section{Immunofluorescent (IF) staining of brain specimens}

The procedure and protocol of IF staining were based on our previous reports [28, 30-33]. In detail, frozen sections ( $4 \mu \mathrm{m}$ thick) was obtained from the brain hemorrhagic area/at risk area of each animal, permeated with $0.5 \%$ Triton X-100, and incubated with antibodies against NueN (1:100, Merck), CD31 (1:100, Abcam), von Willebrand factor (vWF) (1:200, Abcam), CXCR4 (1:100, Invitrogen), CD14 (1:200, Proteintech), and F4/80 (1:100, Santa cruz) at $4{ }^{\circ} \mathrm{C}$ overnight. Alexa Fluor488, Alexa Fluor568, or Alexa Fluor594-conjugated goat anti-mouse or rabbit IgG was used to localize signals. Sections were finally counterstained with DAPI and observed with a fluorescent microscope equipped with epifluorescence (Olympus IX-40). Three brain sections were analyzed for each rat. For quantification, three randomly selected high-power fields (HPFs; 400x for IF study) were analyzed in each section. The mean number of positively-stained cells per HPF for each animal was then be determined by summation of all numbers divided by 9 .

\section{Vessel density in brain infarct zone}

The procedure and protocol for identifying number of small vessels in the brain hemorrhagic area/at risk area were based on our previous reports [31-33]. In detail, staining of small blood vessels was performed with alpha smooth muscle actin (a-SMA) (1:400) as primary antibody at room temperature for 1 hour, followed by washing with PBS thrice. Ten minutes after the addition of anti-mouse-HRP conjugated secondary antibody, the tissue sections were washed with PBS thrice. Then 3,3' diaminobenzidine (DAB) ( $0.7 \mathrm{gm} /$ tablet) (Sigma) will be added, followed by washing with PBS thrice after one minute. Finally, hematoxylin was added as a counter-stain for nuclei, followed by washing twice with PBS after one minute. Three brain sections were analyzed in each rat. For quantification, three randomly selected HPFs (100x) were analyzed in each section. The mean number per HPF for each animal was then be determined by summation of all numbers divided by 9 .

\section{Procedure and protocol of brain magnetic resonance imaging (MRI) for determining the brain hemorrhagic}




\section{volume (BHV)}

The procedure and protocol for brain magnetic resonance imaging (MRI) study were based on our previous report [28]. The MRI was performed at day 28 after IS induction. Briefly, during MRI measurements, mice were anesthetized by $2 \%$ inhalational isoflurane with room air and placed in an MRIcompatible holder (Biospec 94/20, Bruker, Ettingen, Germany). Rectal temperature and respiration were monitored throughout the procedure to ensure normal physiological conditions were maintained. MRI data were collected using a Varian 9.4T animal scanner (Biospec 94/20, Bruker, Ettingen, Germany) with a rat surface array. The MRI protocol consisted of $40 \mathrm{~T} 2$-weighted images. Forty continuous slice locations were imaged with a field-of-view of $30 \mathrm{~mm} \times 30 \mathrm{~mm}$, an acquisition matrix dimension of $256 \times$ 256 and slice thickness of $0.5 \mathrm{~mm}$. The repetition time (TR) and echo time (TE) for each fast spin-echo volume were $4200 \mathrm{~ms}$ and $30 \mathrm{~ms}$, respectively. Custom software, ImageJ (1.43i, NIH, USA), was used to process the region of interest (ROI). Planimetric measurements of images from MRI T2 were performed to calculate the stroke volumes of cortex. Collectively, the BHV was calculated by summation of total coronal sections and then divided the numbers of coronal sections to obtain the means of infarct areas. Additionally, the height of the infarct zone was calculated by summation of the thickness of each coronal sections. Finally, the BHV was obtained by mean of infarct area $\mathrm{x}$ height.

\section{Statistical analysis}

Quantitative data were expressed as means \pm SD. Statistical analysis was adequately performed by ANOVA, followed by Bonferroni multiple-comparison post hoc test. SAS statistical software for Windows version 8.2 (SAS institute, Cary, NC) was utilized. A P value of less than 0.05 was considered statistically significant.

\section{Results}

\section{Identification of hemorrhagic area by day 14 after ICH induction and time courses of neurological function assessed by corner test (Figure 1)}

First, to assess the early stage of intracerebral hemorrhagic zone, the whole brain cross section was stained by TTC by day 14 after ICH induction. The result demonstrated that the intracranial hemorrhagic area was lowest in group 1 (SC), highest in group 2 (ICH only) and significantly lower in group 5 (ICH + HUCDMSC + HBO) than in group $3(\mathrm{ICH}+\mathrm{HUCDMSC})$ and $4(\mathrm{ICH}+\mathrm{HBO})$, but it showed no different between the groups 3 and 4 (Fig. 1-C to $1-\mathrm{H}$ ).

Next, to elucidate the therapeutic impact of HUCDMSC-HBO on preservation of the neurological function (i.e., by corner test), the time courses of corner test (Fig. 1-I) were performed for the animals in each group. The results showed that by day 0 prior to $\mathrm{ICH}$ induction, the neurological function did not differ among the five groups (Fig. 1-J). However, by day 1, the neurological function was significantly impaired in groups 2 to 5 than in group 1, but it showed no different among the groups 2 to 5 (Fig. 1-K). However, 
by days 7,14 and 28 , the neurological function was significantly impaired in group 2 than in group 1 , while significantly progressively improved in groups 3 and 4 and further improved in group 5 (Fig. 1-L to $1-\mathrm{N})$.

\section{Brain hemorrhagic volume and expression of $\mathrm{NeuN+}$ cells in brain hemorrhagic zone by day 28 after ICH induction (Figure 2)}

To further evaluate the anatomical integrity of brain parenchyma, we utilized the brain MRI instrument. As we expected, by day 28 the brain MRI demonstrated that the BHV (Fig. 2-A to 2-E) was lowest in group 1, highest in group 2 and significantly lower in group 5 than in groups 3 and 4 (Fig. 2-F). However, this parameter did not differ between groups 3 and 4 (Fig. 2-F).

To realize the ultrastructure of the brain architecture in these animals, the high manifested IF microscopic analysis was done in the present study. The result revealed that number of NeuN+ cells (Fig. 2-G to 2-K), an indicator of neurons, was highest in group 1, lowest in group 2 and significantly higher in group 5 than in groups 3 and 4 but it showed no difference between groups 3 and 4 (Fig. 2-L).

\section{Impact of HUCDMSCs and HBO therapies on upregulating the angiogenesis capacity in brain parenchyma by day 28 after IHC induction (Figures 3,4 )}

By using the IF microscopic examination, we further determined the angiogenesis ability of HUCDMSCHBO therapy in ischemic zone. The result showed that the expression of CXCR4+ cells (Fig. 3-A to 3-E), an index of endothelial progenitor cells that play a crucial role for angiogenesis, was notably progressively increased from groups 1 to 5 , implicating an intrinsic response to ischemic stimulation that could be more augmented by HUCDMSC-HBO therapy (Fig. 3-F). Additionally, the a-SMA stain demonstrated that the number of small vessels (i.e., defined as the diameter $\leq 25 \mu \mathrm{M}$ ) (Fig. 3-G to 3-K), an indicator of angiogenesis/neovascularization, was highest in group 1, lowest in group 2 and significantly higher in group 5 than in groups 3 and 4 but it did not differ between groups 3 and 4 (Fig. 3-L).

On the next stop, we also utilized the IF microscopic examination to identify the expressions of endothelial-cell surface biomarkers of CD31 (Fig. 4-A to 4-E) and vWF (Fig. 4-G to 4-K), an integrity of endothelial cells. The result showed that these cellular expressions exhibited an identical pattern of number of small vessels among the five groups (Fig. 4-F, Fig. 4-L).

\section{Cellular and molecular level of inflammatory downstream signalingby day 28 after IHC induction (Figures} $5,6,7$ )

It is well recognized that tissue ischemia and necrosis would always induce inflammatory reactions. Thus, we evaluated the protein and cellular level of inflammatory responses. As expected, the cellular expressions of CD14 (Fig. 5-A to 5-E) and F4/80 (Fig. 5-G to 5-K) in brain tissue, two indicators of inflammation, were highest in group 1, lowest in group 1, significantly lower in group 5 than in groups 3 and 4 and significantly lower in group 3 than in group 4 . 
We then returned to see the molecular level of inflammation. Consistently, the Western blot analysis showed that the protein expressions of HMGB1, TLR-2, TLR-4, MyD88 and TRAF6 (Fig. 6-A to 6-E), five indices of upstream inflammatory signaling, displayed a similar pattern of CD14+ cells among the five groups. Additionally, the protein expressions of p-NF-KB, IFN-y, IL-1ß and TNF-a (Fig. 7-B to 7-E), four indicators of downstream inflammatory signaling, also exhibited a similar pattern of CD14+ cell, whereas the protein expression of IKB-ß (Fig. 7-A), another indicator of downstream signaling, displayed an opposite pattern of CD14+ cells among the five groups.

\section{Protein level of oxidative stress and autophagic biomarkers by day 28 after IHC induction (Figure 8 )}

It is also well recognized that tissue ischemia and necrosis always elicit oxidative stress which, in turn, induces apoptosis and upregulated the autophagy. Based on this concept, we performed Western blot analysis. The result demonstrated that the protein expressions of NOX-1, NOX-2 and oxidized protein (Fig. 3-A to $3-C)$, three indices of oxidative stress, were lowest in group 1, highest in group 2 and significantly lower in group 5 than in groups 3 and 4, but they showed no difference between groups 3 and 4 . Additionally, the protein expressions of cleaved caspase 3 (Fig. 3-D), and indicator of apoptosis, was lowest in group 1, highest in group 2, significantly lower in group 5 than in groups 3 and 4 and significantly lower in group 3 than in group 4. On the other hand, the cleaved PARP (Fig. 3-E), another indicator of apoptosis and the ratio of LC3B-II/LC3B-I (Fig. 3-F), an indicator of autophagic biomarker, exhibited an identical pattern of oxidative stress among the five groups.

\section{Discussion}

This study which investigated the therapeutic impact of HUCDMSC-HBO on ICH rodent yielded several preclinical implications. First, HUCDMSC or HBO therapy substantially reduced the brain hemorrhagic area (i.e., estimated by TCC stain at early stage of $\mathrm{ICH}$ ) and brain hemorrhagic volume (i.e., estimated by brain MRI at day 28 after ICH) and remarkably preserved neurological function (i.e., estimated by corner test). Second, the extensive woks were done in the present study and found that inflammatory signaling and oxidative stress could be the mainly underlying mechanism for brain damage after ICH induction. Third, therapeutic impact of HBO was comparable with HUCDMSC for protecting the brain architecture against hemorrhagic damage. Finally, the combined HUCDMSC-HBO therapy was superior to either one alone on protecting the brain volume and neurological function in setting of $\mathrm{ICH}$.

The effective treatment of severe ICH is still lacking. This could explain for why this disease entity is still a high cause of death and disability in adults worldwide [1-3]. Interestingly, our recent studies [27, 28] have shown that exogenous MSCs treatment for rodent IS or brain hemorrhage was safe and without immune rejection. Of importance was that these MSCs therapy effectively protected the brain from ischemic related/hemorrhagic relayed injury $[27,28]$. Additionally, our other recent studies have shown that HBO therapy effectively preserved the brain infarct volume and neurological function in rat after IS $[30,34]$. The most important finding in the present study was that as compared with ICH group, the brain hemorrhagic volume and brain hemorrhagic zone were significantly preserved in $\mathrm{IHC}$ animals treated by 
HUCDMSCs and HBO. Of distinctive importance was that combined HUCDMSCs and HBO offer additional benefit than single one therapy alone on preserving the brain architecture and neurological integrity after $\mathrm{ICH}$ attack. Our findings, in addition to strengthening the findings of previous studies [27, 28, 30,34], could, at least in part, explain for why the neurological function of $\mathrm{ICH}$ animals was greatly improved with HUCDMSC-HBO therapy.

It is well documented that the mechanical damage is always immediately elicited after ICH which commonly wrecks the brain tissue as it enlarges [2]. This, is frequently associated with the pressure created enough by blood and surrounding brain edema, resulting in a life-threatening situation [4]. The secondary damage is caused by cytotoxicity of blood [5, 6], impaired calcium homeostasis [7], excitotoxicity from excitatory neurotransmitters/glial cells [8,9] and upregulation of oxidative stress and inflammation [6,9-14]. Additionally, an association between the upregulation of inflammation and oxidative stress and organ damage in setting of ischemia or ischemia-reperfusion injury have been extensively investigated by previous studies [19-24]. An essential finding in the present study was that as compared with SC group, the brain hemorrhagic volume (i.e., caused mechanical damage) and the inflammatory downstream signaling and oxidative stress (i.e., caused by cellular and molecular perturbations) were remarkably upregulated in $\mathrm{ICH}$ animals (refer to Fig. 9). Our findings, in addition to being consistent with the findings of the previous studies [2, 4-7, 9-14, 19-24], could explain for why much higher apoptosis autophagic phenomenon and the unfavorable outcomes developed in the IHC animals, and suggest that inflammatory signaling/oxidative stress plaid a ultimately crucial role on damage (i.e., second damage) of the brain architecture and neuron integrity after $\mathrm{ICH}$.

The mechanisms for why MSCs and HBO therapy effectively protect the cells/tissues and organs from any ischemia-related damage always elicit high interest from scientists [35, 36]. Currently, abundant data have shown that anti-inflammation, immunomodulation and tissue regeneration as well as cytokine/paracrine effects could be the main mechanisms for MSCs therapy on safeguarding the cells/tissues and organs far away from inflammatory and ischemic related damage $[27,28,36]$. Our findings demonstrated even xenogenic HUCDMSCs (i.e., from human derived MSC to rodent) was still safe without any immune reaction and offered additional benefit to IHC rats (refer to Fig. 9). In this way, our findings corroborated with the findings of previous studies $[27,28,36]$. On the other hand, the mechanistic basis of HBO therapy on protecting the ischemic cells/tissues and organs from ischemic damage has been emphasized mainly due to upregulation of circulating levels of endothelial progenitor cells/MSCs, an increase in vascular permeability, high content of oxygen in ischemic tissues and antiapoptotic and anti-inflammatory effects $[27,28,36]$. These findings $[27,28,36]$ could explain for why a synergic effect of combined HUCDMSC + HBO therapy on reducing the brain hemorrhagic volume and improving the neurological function to be identified in the present study.

We proposed the therapeutic regimen of the multiple dosages of HUCDMSCs (i.e., like the regimen of antibiotics to be utilized for treatment the bacterial-induced sepsis in our daily clinical practice) could be the key to success for remarkable preservation the brain architecture and neurological function. Interestingly, our previous [36] and recent [37] studies have shown that early and multiple dosages of 
stem cell administration was the crucial role on reducing the post-heart transplant acute rejection [37] and preservation of the neurological function after acute ischemic stroke [38] in rodent. In this way, the results of the present study, in addition to being comparable with the findings of our previous [36] and recent [37] studies, highlight that this regimen could be seriously considered in our future stem cell therapy for the patients.

\section{Study limitations}

This study has limitations. Despite a lot of works had been done in the present study, the exact mechanisms of combined HUCDMSCs and HBO on preserving the brain architecture and neurological function were still not fully investigated. Based on the results of the present study, Fig. 9 illustrated the proposed mechanism for how the HUCDMSC + HBO therapy on improving the outcomes after IHC in rodent. Second, although the short-term outcomes (i.e., at a study period of 28 days) were attractive and promising, the long-term impact of such kind of therapy remains uncertain.

\section{Conclusions}

In conclusion, the results of the present study demonstrated that combined HUCDMSC and HBO therapy offered a synergic effect on protecting the macro- and micro-ultrastructure of the brain and preserving neurological integrity in rat after $\mathrm{ICH}$.

\section{Abbreviations}

a-SMA: alpha smooth muscle actin; BHV: brain-hemorrhagic volume; DAMP: damage-associated molecular patterns; HBO: hyperbaric oxygen; HMGB1: high mobility group box 1; HUCDMSC: human umbilical cord-derived mesenchymal stem cell; ICH: intracerebral hemorrhage; INF: interferon; MDR88: myeloid differentiation primary response 88; NF-kB: nuclear factor -кB; PARP: poly (ADP-ribose) polymerase; TLR: toll-like receptor; TNF: tumor necrosis factor; TRAF: tumor necrosis factor associated factor; vWF: von Willebrand factor.

\section{Declarations}

\section{Ethics approval and consent to participate:}

All animal procedures were approved by the Institute of Animal Care and Use Committee at Kaohsiung Chang Gung Memorial Hospital (Affidavit of Approval of Animal Use Protocol No. 2018032102) and performed in accordance with the Guide for the Care and Use of Laboratory Animals, 8th edition (NIH publication No. 85-23, National Academy Press, Washington, DC, USA, revised 2011).

\section{Consent for publication:}

Not applicable. 
Availability of data and materials:

The data that support the findings of this study are available from the corresponding authors upon reasonable request.

\section{Competing interests:}

All authors have read the journal's policy on disclosure of potential conflicts of interest and the journal's authorship agreement. The authors declare that they have no conflicts of interest. The article has been reviewed by and approved by all named authors.

\section{Funding:}

This study was supported by a program grant from Chang Gung Memorial Hospital, Chang Gung University (Grant no. CMRPG8H0571).

\section{Author contributions:}

Hon-Kan Yip, Kun-Chen Lin, and Kuan-Hung Chen conceptualized the study and designed the experiments. Pei-Hsun Sung and John Y. Chiang acquired, analyzed and interpreted data. Tsung-Cheng Yin and Re-Wen Wu were responsible for the laboratory assays and troubleshooting. Hon-Kan Yip, KunChen Lin, and Kuan-Hung Chen drafted, reviewed and edited the manuscript

\section{Acknowledgement:}

This study was supported by a program grant from Chang Gung Memorial Hospital, Chang Gung University (Grant no. CMRPG8H0571).

\section{References}

1. Qureshi Al, Tuhrim S, Broderick JP, Batjer HH, Hondo H, Hanley DF. Spontaneous intracerebral hemorrhage. N Engl J Med. 2001;344:1450-60.

2. Qureshi Al, Mendelow AD, Hanley DF. Intracerebral haemorrhage. Lancet. 2009;373:1632-44.

3. Chan CL, Ting HW, Huang HT. The incidence, hospital expenditure, and, 30 day and 1 year mortality rates of spontaneous intracerebral hemorrhage in Taiwan. J Clin Neurosci. 2014;21:91-4.

4. Gross BA, Jankowitz BT, Friedlander RM. Cerebral Intraparenchymal Hemorrhage: A Review. JAMA. 2019;321:1295-303.

5. Wagner KR, Sharp FR, Ardizzone TD, Lu A, Clark JF. Heme and iron metabolism: role in cerebral hemorrhage. J Cereb Blood Flow Metab. 2003;23:629-52.

6. Xi G, Keep RF, Hoff JT. Mechanisms of brain injury after intracerebral haemorrhage. Lancet Neurol. 2006;5:53-63. 
7. Tymianski $\mathrm{M}$, Tator $\mathrm{CH}$. Normal and abnormal calcium homeostasis in neurons: a basis for the pathophysiology of traumatic and ischemic central nervous system injury. Neurosurgery. 1996;38:1176-95.

8. Qureshi Al, Ali Z, Suri MF, Shuaib A, Baker G, Todd K, Guterman LR, Hopkins LN. Extracellular glutamate and other amino acids in experimental intracerebral hemorrhage: an in vivo microdialysis study. Crit Care Med. 2003;31:1482-9.

9. Aronowski J, Zhao X. Molecular pathophysiology of cerebral hemorrhage: secondary brain injury. Stroke. 2011;42:1781-6.

10. Gong C, Hoff JT, Keep RF. Acute inflammatory reaction following experimental intracerebral hemorrhage in rat. Brain Res. 2000;871:57-65.

11. Hickenbottom SL, Grotta JC, Strong R, Denner LA, Aronowski J. Nuclear factor-kappaB and cell death after experimental intracerebral hemorrhage in rats. Stroke. 1999;30:2472-7; discussion 7-8.

12. Xue M, Del Bigio MR. Intracerebral injection of autologous whole blood in rats: time course of inflammation and cell death. Neurosci Lett. 2000;283:230-2.

13. Tang J, Liu J, Zhou C, Ostanin D, Grisham MB, Neil Granger D, Zhang JH. Role of NADPH oxidase in the brain injury of intracerebral hemorrhage. J Neurochem. 2005;94:1342-50.

14. Wang J, Fields J, Zhao C, Langer J, Thimmulappa RK, Kensler TW, Yamamoto M, Biswal S, Dore S. Role of Nrf2 in protection against intracerebral hemorrhage injury in mice. Free Radic Biol Med. 2007;43:408-14.

15. Stonesifer C, Corey S, Ghanekar S, Diamandis Z, Acosta SA, Borlongan CV. Stem cell therapy for abrogating stroke-induced neuroinflammation and relevant secondary cell death mechanisms. Prog Neurobiol. 2017;158:94-131.

16. Werner C, Engelhard K. Pathophysiology of traumatic brain injury. Br J Anaesth. 2007;99:4-9.

17. Bennett MH, Trytko B, Jonker B. Hyperbaric oxygen therapy for the adjunctive treatment of traumatic brain injury. Cochrane Database Syst Rev. 2012;12:CD004609.

18. Gonzales-Portillo B, Lippert T, Nguyen H, Lee JY, Borlongan CV. Hyperbaric oxygen therapy: A new look on treating stroke and traumatic brain injury. Brain Circ. 2019;5:101-5.

19. Sun CK, Yen CH, Lin YC, Tsai TH, Chang LT, Kao YH, Chua S, Fu M, Ko SF, Leu S, et al. Autologous transplantation of adipose-derived mesenchymal stem cells markedly reduced acute ischemiareperfusion lung injury in a rodent model. J Transl Med. 2011;9:118.

20. Yip HK, Chang YC, Wallace CG, Chang LT, Tsai TH, Chen YL, Chang HW, Leu S, Zhen YY, Tsai CY, et al. Melatonin treatment improves adipose-derived mesenchymal stem cell therapy for acute lung ischemia-reperfusion injury. J Pineal Res. 2013;54:207-21.

21. Chen HH, Lin KC, Wallace CG, Chen YT, Yang CC, Leu S, Chen YC, Sun CK, Tsai TH, Chen YL, et al. Additional benefit of combined therapy with melatonin and apoptotic adipose-derived mesenchymal stem cell against sepsis-induced kidney injury. J Pineal Res. 2014;57:16-32. 
22. Chen YT, Chiang HJ, Chen CH, Sung PH, Lee FY, Tsai TH, Chang CL, Chen HH, Sun CK, Leu S, et al. Melatonin treatment further improves adipose-derived mesenchymal stem cell therapy for acute interstitial cystitis in rat. J Pineal Res. 2014;57:248-61.

23. Chang CL, Sung PH, Sun CK, Chen CH, Chiang HJ, Huang TH, Chen YL, Zhen YY, Chai HT, Chung SY, et al. Protective effect of melatonin-supported adipose-derived mesenchymal stem cells against small bowel ischemia-reperfusion injury in rat. J Pineal Res. 2015;59:206-20.

24. Lee FY, Chen KH, Wallace CG, Sung PH, Sheu JJ, Chung SY, Chen YL, Lu HI, Ko SF, Sun CK, et al. Xenogeneic human umbilical cord-derived mesenchymal stem cells reduce mortality in rats with acute respiratory distress syndrome complicated by sepsis. Oncotarget. 2017;8:45626-42.

25. Le Blanc K, Tammik L, Sundberg B, Haynesworth SE, Ringden O. Mesenchymal stem cells inhibit and stimulate mixed lymphocyte cultures and mitogenic responses independently of the major histocompatibility complex. Scand J Immunol. 2003;57:11-20.

26. Maumus M, Guerit D, Toupet K, Jorgensen C, Noel D. Mesenchymal stem cell-based therapies in regenerative medicine: applications in rheumatology. Stem Cell Res Ther. 2011;2:14.

27. Chen KH, Lin KC, Wallace CG, Li YC, Shao PL, Chiang JY, Sung PH, Yip HK. Human induced pluripotent stem cell-derived mesenchymal stem cell therapy effectively reduced brain infarct volume and preserved neurological function in rat after acute intracranial hemorrhage. Am J Transl Res. 2019;11:6232-48.

28. Chen KH, Chen CH, Wallace CG, Yuen CM, Kao GS, Chen YL, Shao PL, Chen YL, Chai HT, Lin KC, et al. Intravenous administration of xenogenic adipose-derived mesenchymal stem cells (ADMSC) and ADMSC-derived exosomes markedly reduced brain infarct volume and preserved neurological function in rat after acute ischemic stroke. Oncotarget. 2016;7:74537-56.

29. Lu Q, Huang L, ZhuGe Q. A rat model of intracerebral hemorrhage induced by collagenase IV. Bioprotocol. 2015;5:e1541.

30. Lin KC, Chen KH, Wallace CG, Chen YL, Ko SF, Lee MS, Yip HK. Combined Therapy With Hyperbaric Oxygen and Melatonin Effectively Reduce Brain Infarct Volume and Preserve Neurological Function After Acute Ischemic Infarct in Rat. J Neuropathol Exp Neurol. 2019;78:949-60.

31. Chen YL, Tsai TH, Wallace CG, Chen YL, Huang TH, Sung PH, Yuen CM, Sun CK, Lin KC, Chai HT, et al. Intra-carotid arterial administration of autologous peripheral blood-derived endothelial progenitor cells improves acute ischemic stroke neurological outcomes in rats. Int J Cardiol. 2015;201:668-83.

32. Yuen CM, Chung SY, Tsai TH, Sung PH, Huang TH, Chen YL, Chen YL, Chai HT, Zhen YY, Chang MW, et al. Extracorporeal shock wave effectively attenuates brain infarct volume and improves neurological function in rat after acute ischemic stroke. Am J Transl Res. 2015;7:976-94.

33. Chen HH, Chen YT, Yang CC, Chen KH, Sung PH, Chiang HJ, Chen CH, Chua S, Chung SY, Chen YL, et al. Melatonin pretreatment enhances the therapeutic effects of exogenous mitochondria against hepatic ischemia-reperfusion injury in rats through suppression of mitochondrial permeability transition. J Pineal Res. 2016;61:52-68. 
34. Hsu SL, Yin TC, Shao PL, Chen KH, Wu RW, Chen CC, Lin PY, Chung SY, Sheu JJ, Sung PH, et al. Hyperbaric oxygen facilitates the effect of endothelial progenitor cell therapy on improving outcome of rat critical limb ischemia. Am J Transl Res. 2019;11:1948-64.

35. Lin PY, Sung PH, Chung SY, Hsu SL, Chung WJ, Sheu JJ, Hsueh SK, Chen KH, Wu RW, Yip HK. Hyperbaric Oxygen Therapy Enhanced Circulating Levels of Endothelial Progenitor Cells and Angiogenesis Biomarkers, Blood Flow, in Ischemic Areas in Patients with Peripheral Arterial Occlusive Disease. J Clin Med. 2018;7:

36. Yip HK, Lee MS, Sun CK, Chen KH, Chai HT, Sung PH, Lin KC, Ko SF, Yuen CM, Liu CF, et al.

Therapeutic effects of adipose-derived mesenchymal stem cells against brain death-induced remote organ damage and post-heart transplant acute rejection. Oncotarget. 2017;8:108692-711.

37. Lin KC, Chai HT, Chen KH, Sung PH, Chiang JY, Shao PL, Huang CR, Li YC, Ko SF, Yip HK. Intra-carotid arterial transfusion of circulatory-derived autologous endothelial progenitor cells in rodent after ischemic stroke-evaluating the impact of therapeutic time points on prognostic outcomes. Stem Cell Res Ther. 2020;11:219.

\section{Figures}
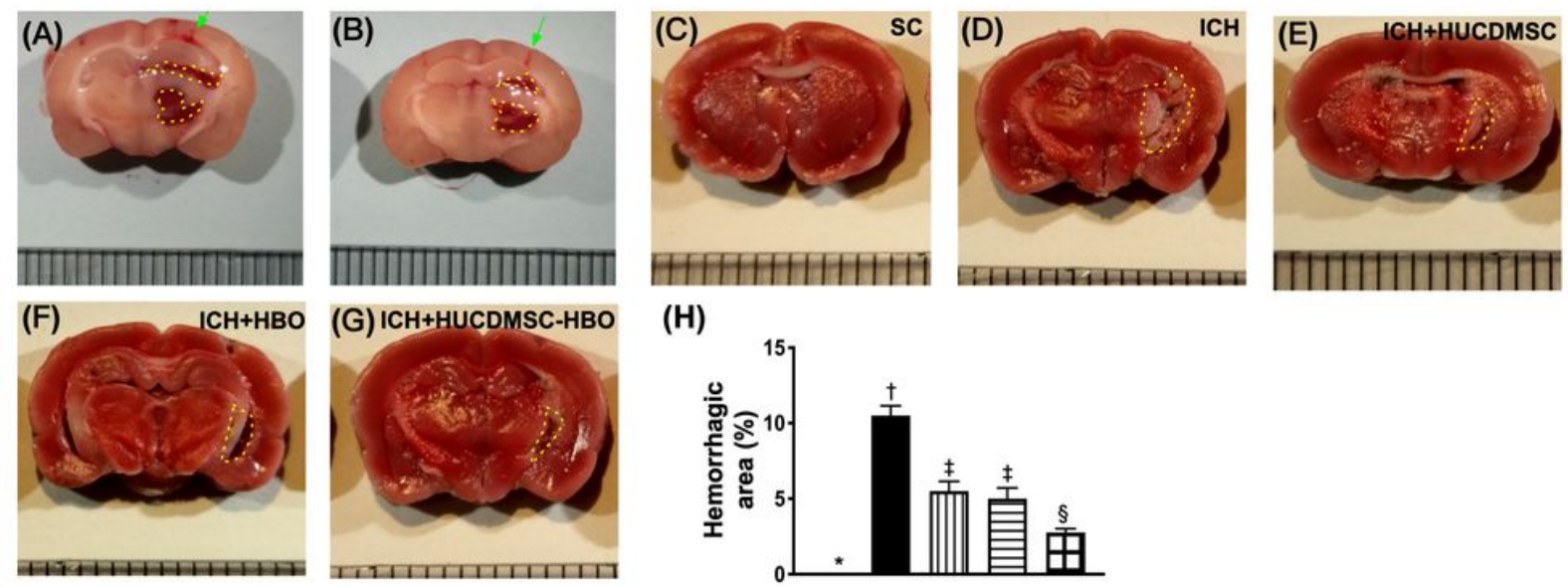

(H)
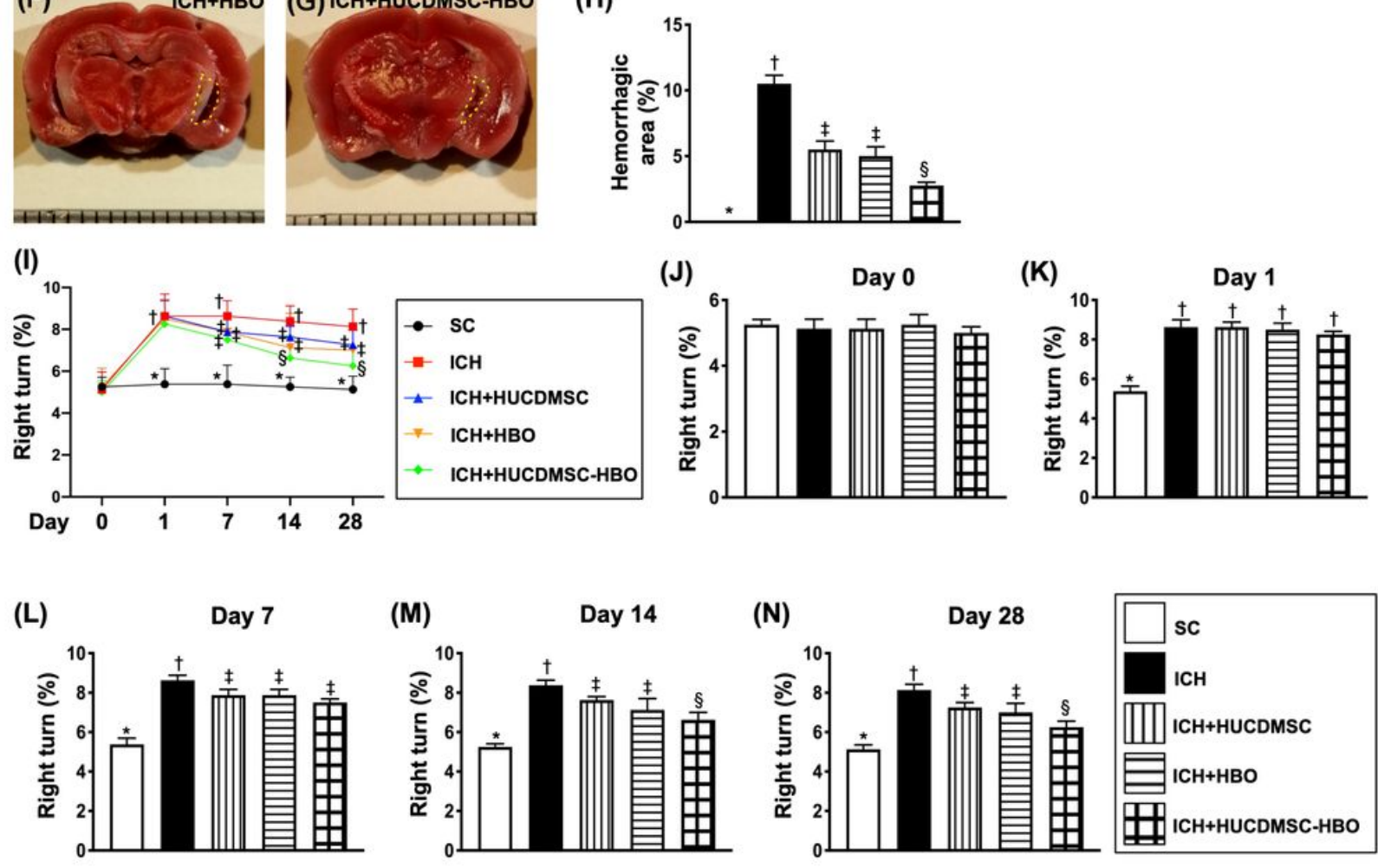


\section{Figure 1}

Intracerebral hemorrhagic area by day 14 after $\mathrm{ICH}$ induction and time courses of neurological function assessed by corner test A and B) Illustrating two whole cross sections of brain which demonstrated intracranial injection of type IV collagenase successfully induced intracranial hemorrhage (red color) (yellow dotted lines). Green arrow indicated the needle track. C to G) Illustrating the triphenyltetrazolium chloride (TTC) (100x) of whole brain cross section for identification of percentage of intracerebral hemorrhagic area (the yellow dotted line indicated the boundary of the area) by day 14 after ICH induction $(n=4) . H)$ Statistical analysis of summated (four brain-cross sections in each animal) intracerebral hemorrhagic area, * vs. other groups with different symbols $(\dagger, \ddagger, \S), p<0.001$. I) Illustrating the corner test for determining neurological function among days $0,1,7,14$ and 28 after acute $\mathrm{ICH}$ induction. J) Statistical analysis of neurological function (i.e., by corner test) by day $0, p>0.5$. K) Statistical analysis of neurological function day 1 , * vs. $\dagger, p<0.001$. L) Statistical analysis by day 7 , * vs. other groups with different symbols $(\dagger, \ddagger), p<0.0001$. M) Statistical analysis by day $14,{ }^{*}$ vs. other groups with different symbols $(\dagger, \ddagger, \S), p<0.001$. N) Statistical analysis by day $28, * v s$. other groups with different symbols $(\dagger, \ddagger, \S), p<0.0001 . n=6$ for each group. All statistical analyses were performed by oneway ANOVA, followed by Bonferroni multiple comparison post hoc test. Symbols $(*, \dagger, \ddagger, \S)$ indicate significance (at 0.05 level). SC = sham-operated control; ICH = intracerebral hemorrhage; $\mathrm{HUCDMSC}=$ human umbilical cord-derived mesenchymal stem cell; $\mathrm{HBO}=$ hyperbaric oxygen. 

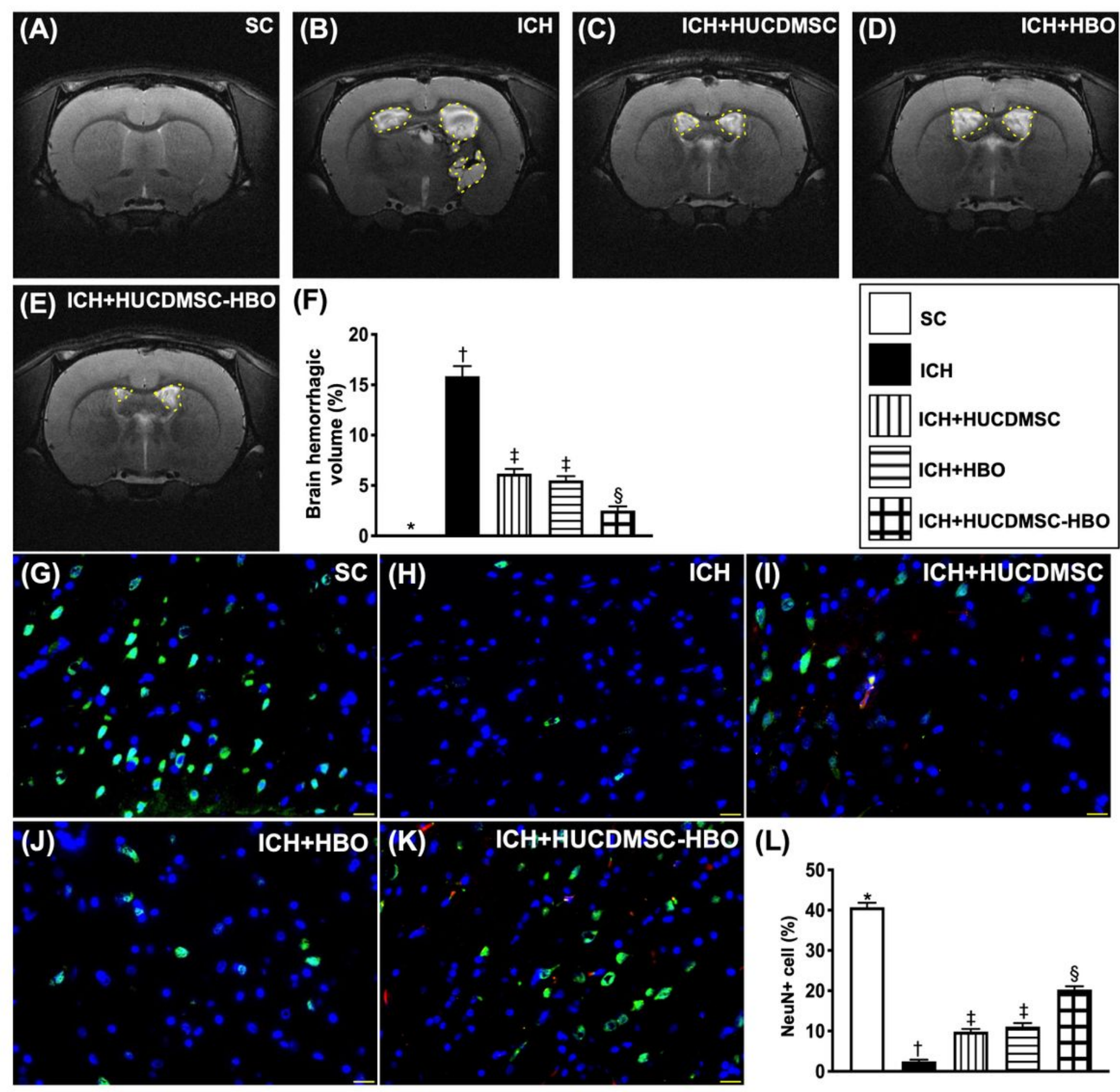

Figure 2

Brain hemorrhagic volume and expression of NeuN+ cells in brain hemorrhagic zone by day 28 after ICH induction $\mathrm{A}$ to $\mathrm{E}$ ) Illustrating the brain magnetic resonance imaging (MRI) finding for identification of brain hemorrhagic volume (white color) (red dotted-line area). F) Analytical result of brain hemorrhagic volume (i.e., the percentage of the whole brain volume), * vs. other groups with different symbols $(\dagger, \ddagger, \S)$, $\mathrm{p}<0.0001$. G to K) Illustrating the immunofluorescent microscopic finding (400x) for identification of expression of NeuN+ cells (green color). Red color in (I) and (K) indicated the implanted cells. L) 
Analytical result of number of NeuN+ cells, * vs. other groups with different symbols $(t, \ddagger, \S), p<0.0001$. Scale bar in right lower corner represent $20 \mu \mathrm{m}$. All statistical analyses were performed by one-way ANOVA, followed by Bonferroni multiple comparison post hoc test $(n=6$ for each group). Symbols $(*, t, \neq$, $\S$ ) indicate significance (at 0.05 level). SC = sham-operated control; $\mathrm{ICH}=$ intracerebral hemorrhage; HUCDMSC = human umbilical cord-derived mesenchymal stem cell; $\mathrm{HBO}=$ hyperbaric oxygen.

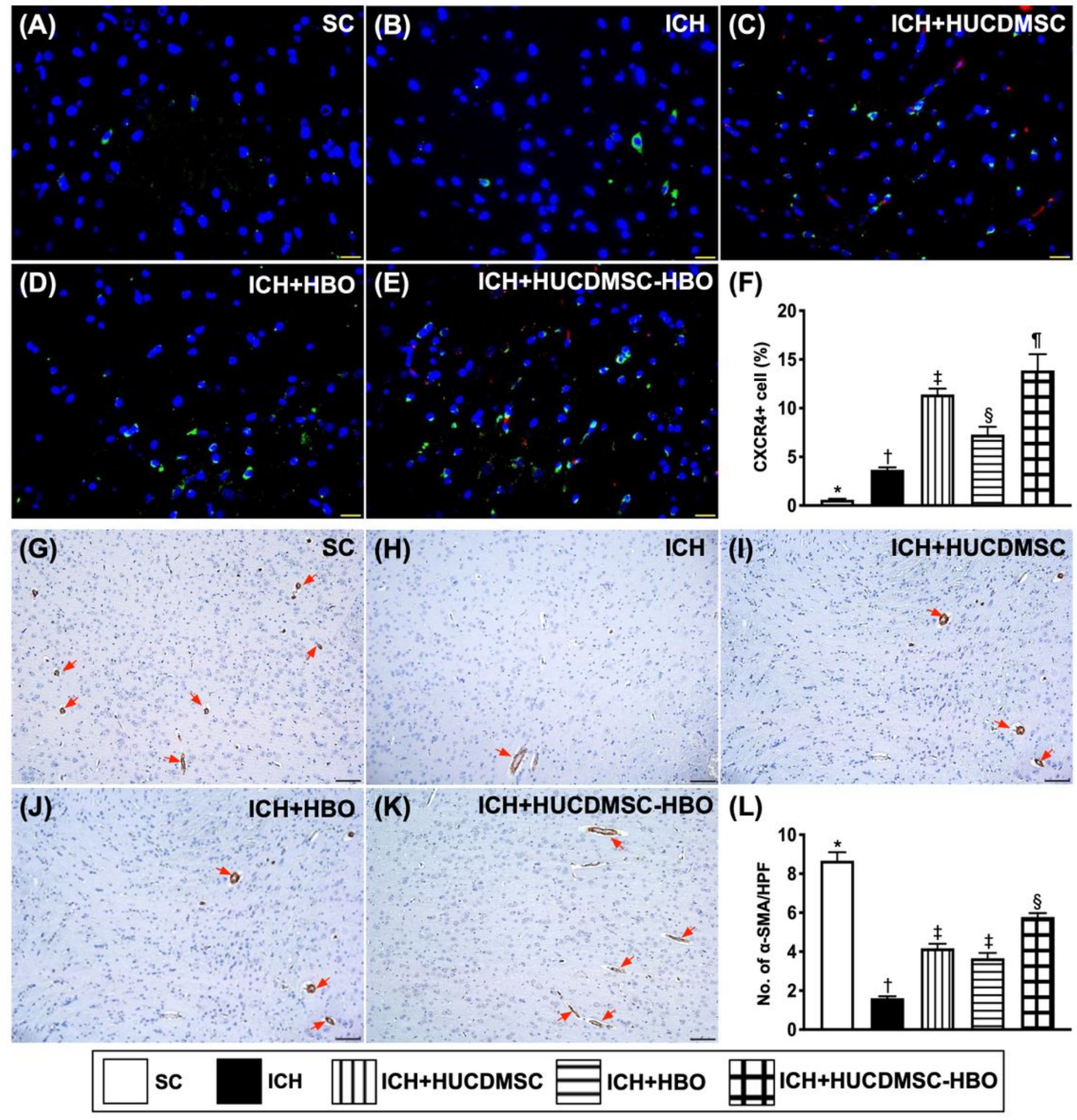

Figure 3 
HUCDMSCs and HBO therapies enhanced angiogenesis in brain parenchyma by day 28 after ICH induction $A$ to $E$ ) Showing the immunofluorescent microscopic finding (400x) for identification of cellular expression of CXCR4 (green color). Red color in (C) and (E) indicated the implanted cells. F) Analytical result of number of CXCR4+ cells, * vs. other groups with different symbols $(t, \ddagger, \S, \mathbf{q}), p<0.0001$. Scale bar in right lower corner represent $20 \mu \mathrm{m}$. G to K) Showing the alpha smooth muscle actin (a-SMA) stain (100x) for identifying the small vessels (gray color) in brain parenchyma (red arrows). Analytical result of number of small vessels (i.e., diameter $\leq 25 \mu \mathrm{M})$ ) * vs. other groups with different symbols $(\dagger, \ddagger, \S)$, $p<0.0001$. Scale bar in right lower corner represent $20 \mu \mathrm{m}$. All statistical analyses were performed by oneway ANOVA, followed by Bonferroni multiple comparison post hoc test ( $\mathrm{n}=6$ for each group). Symbols (*, $\dagger, \ddagger, \S, 9$ ) indicate significance (at 0.05 level). SC = sham-operated control; ICH = intracerebral hemorrhage; $\mathrm{HUCDMSC}=$ human umbilical cord-derived mesenchymal stem cell; $\mathrm{HBO}=$ hyperbaric oxygen. 


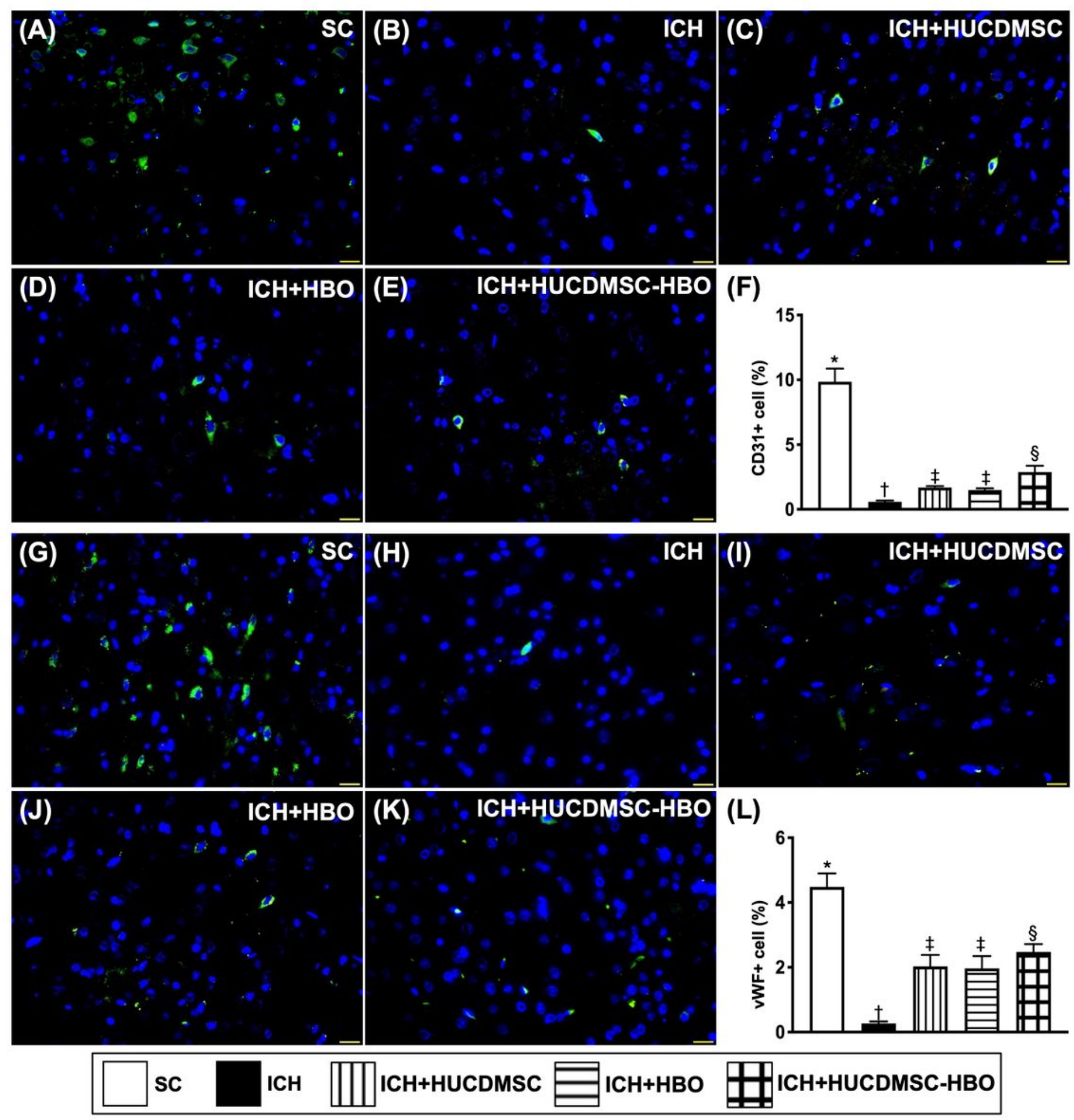

Figure 4

HUCDMSCs and HBO therapies enhanced the expressions of endothelial cell surface markers in brain parenchyma by day 28 after ICH induction A to E) Demonstrating the immunofluorescent (IF) microscopic finding (400x) for identification of CD31+ cells (green color). F) Analytical result of number of CD31+ cells, * vs. other groups with different symbols $(\dagger, \ddagger, \S), p<0.0001$. G to K) Demonstrating the IF microscopic finding (400x) for identification of von Willebrand factor (vWF)+ cells (green color). L) 
Analytical result of number of vWF+ cells, * vs. other groups with different symbols $(\dagger, \ddagger, \S), p<0.0001$. Scale bar in right lower corner represent $20 \mu \mathrm{m}$. All statistical analyses were performed by one-way ANOVA, followed by Bonferroni multiple comparison post hoc test $(n=6$ for each group). Symbols $(*,+, \neq$, $\S$ ) indicate significance (at 0.05 level). SC = sham-operated control; $\mathrm{ICH}=$ intracerebral hemorrhage; HUCDMSC = human umbilical cord-derived mesenchymal stem cell; HBO = hyperbaric oxygen.

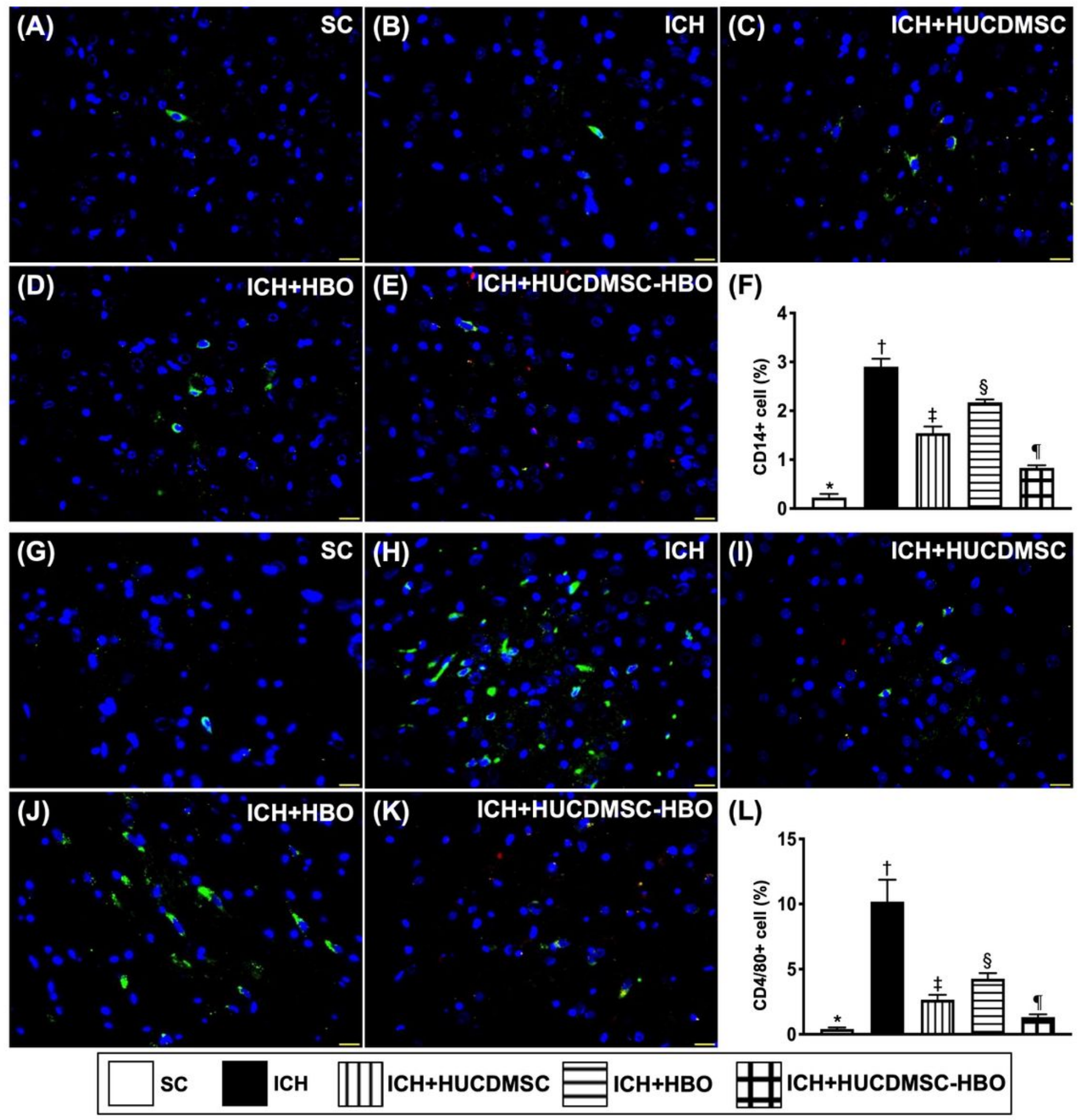

Figure 5 
Inflammatory cell infiltration in brain parenchyma by day 28 after ICH induction A to E) Illustrating the immunofluorescent (IF) microscopic finding (400x) for cellular expression of CD14 (green color). Red color indicated the implanted cells. F) Analytical result of number of CD14+ cells, * vs. other groups with different symbols $(\dagger, \ddagger, \S), p<0.0001$. G to K) Illustrating the IF microscopic finding (400x) for cellular expression of F4/80 (green color). L) Analytical result of number of CD4/80+ cells, * vs. other groups with different symbols $(\dagger, \ddagger, \S), p<0.0001$. Scale bar in right lower corner represent $20 \mu \mathrm{m}$. All statistical analyses were performed by one-way ANOVA, followed by Bonferroni multiple comparison post hoc test ( $n=6$ for each group). Symbols $\left({ }^{*}, t, \neq, \S\right)$ indicate significance (at 0.05 level). SC = sham-operated control; $\mathrm{ICH}=$ intracerebral hemorrhage; $\mathrm{HUCDMSC}=$ human umbilical cord-derived mesenchymal stem cell; HBO = hyperbaric oxygen.

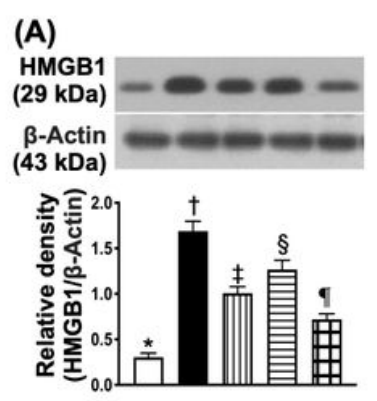

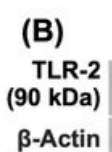

(43 kDa)

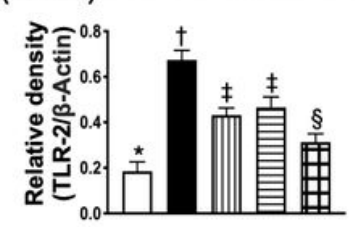

(C)

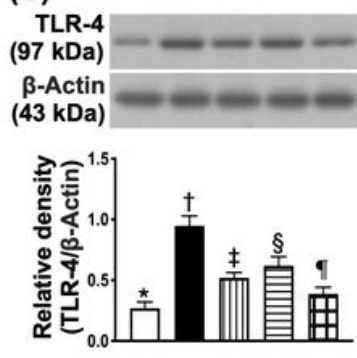

(D)

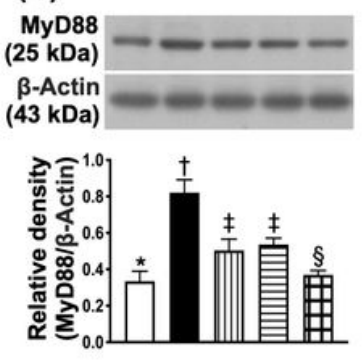

(E)

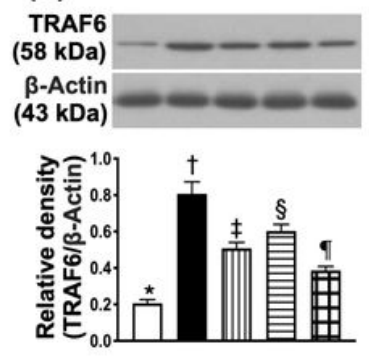

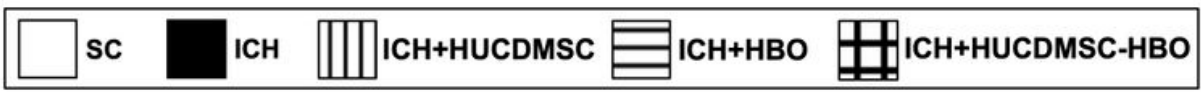

\section{Figure 6}

Protein expressions of upstream inflammatory signaling in brain parenchyma by day 28 after ICH induction A) Protein expression of high mobility group box 1 (HMGB1), * vs. other groups with different symbols $(t, \neq, \S, \mathbf{q}), p<0.0001$. B) Protein expression of toll-like receptor 2 (TLR2), * vs. other groups with different symbols $(t, \neq, \S), p<0.0001$. C) Protein expression of TLR-4, *vs. other groups with different symbols $(\dagger, \neq, \S, \mathbf{q}), p<0.0001$. D) Protein expression of myeloid differentiation primary response 88 (MyD88), * vs. other groups with different symbols $(t, \ddagger, \S), p<0.001$. E) Protein expression of TNF receptor associated factor 6 (TRAF6), * vs. other groups with different symbols $(\dagger, \neq, \S, \mathbf{q}), p<0.001$. All statistical analyses were performed by one-way ANOVA, followed by Bonferroni multiple comparison post hoc test ( $n=6$ for each group). Symbols $\left({ }^{*},+, \neq, \S, 9\right)$ indicate significance (at 0.05 level). SC = shamoperated control; $\mathrm{ICH}=$ intracerebral hemorrhage; $\mathrm{HUCDMSC}=$ human umbilical cord-derived mesenchymal stem cell; $\mathrm{HBO}=$ hyperbaric oxygen . 
(A)

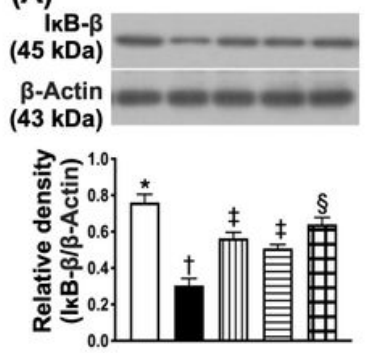

(B)

p-NF-kB

B-Actin

(43 kDa)

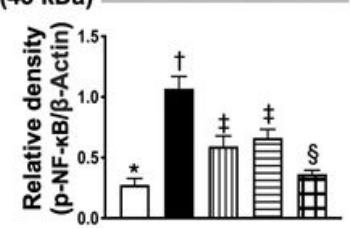

(C)
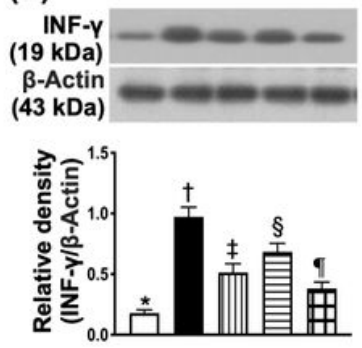

(D)
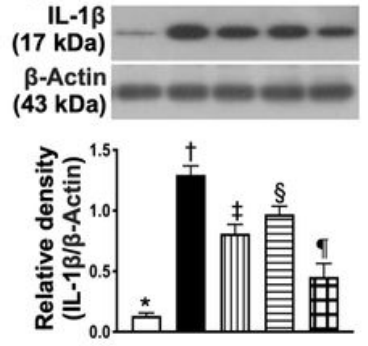

(E)

TNF- $\alpha$
$(25 \mathrm{kDa})$

B-Actin

(43 kDa)

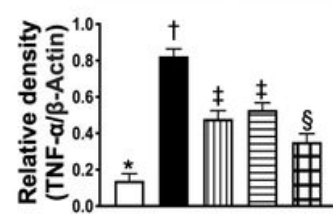

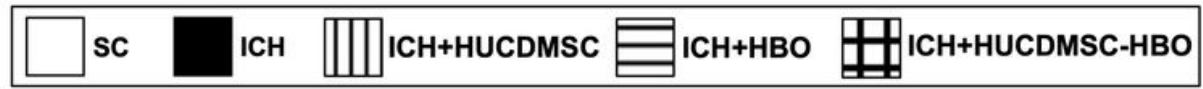

Figure 7

Protein expressions of downstream inflammatory signaling in brain parenchyma by day 28 after $\mathrm{ICH}$ induction A) Protein expression of nuclear factor of kappa light polypeptide gene enhancer in B-cells inhibitor beta (IKB-ß), * vs. other groups with different symbols $(\dagger, \neq, \S), p<0.001$. B) Protein expression of phosphorylated (p) nuclear factor ( $p-N F)-\kappa B$, * vs. other groups with different symbols $(\dagger, \ddagger, \S), p<0.001$.

C) Protein expression of interferon (INF) $-\gamma$, * vs. other groups with different symbols $(\dagger, \neq, \S, q), p<0.001$.

D) Protein expression of interleukin (IL)-1ß, *vs. other groups with different symbols $(\dagger, \ddagger, \S, \mathbf{q}), p<0.001$.

E) Protein expression of tumor necrosis factor (TNF)- $a$, * vs. other groups with different symbols $(\dagger, \ddagger, \S)$, $p<0.001$. All statistical analyses were performed by one-way ANOVA, followed by Bonferroni multiple comparison post hoc test ( $\mathrm{n}=6$ for each group). Symbols $\left({ }^{*},+, \ddagger, \S, 9\right)$ indicate significance (at 0.05 level). $\mathrm{SC}$ = sham-operated control; $\mathrm{ICH}$ = intracerebral hemorrhage; HUCDMSC = human umbilical cord-derived mesenchymal stem cell; $\mathrm{HBO}$ = hyperbaric oxygen.

(A)
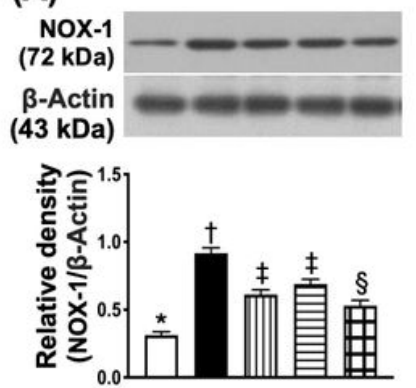

(D)
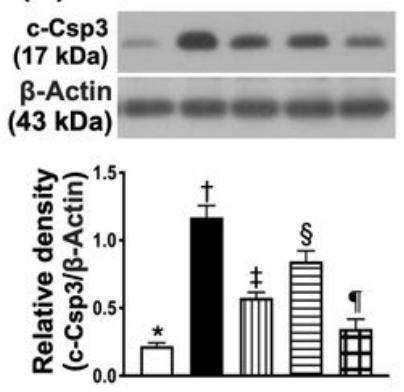

(B)

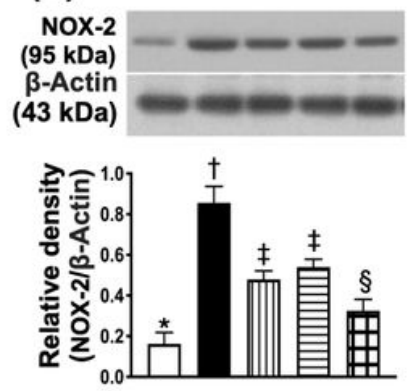

(E)

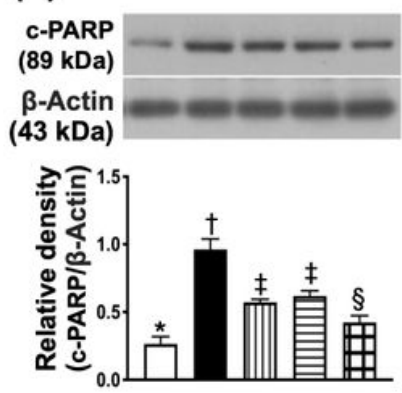

(C)
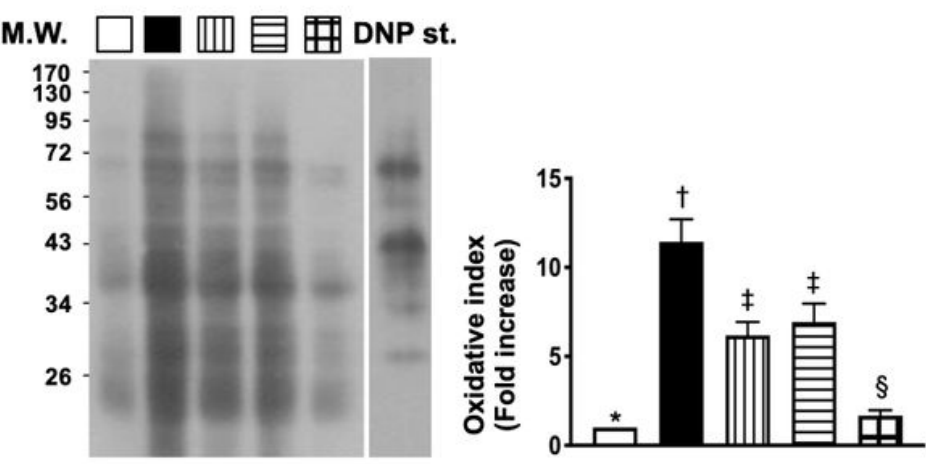

(F)

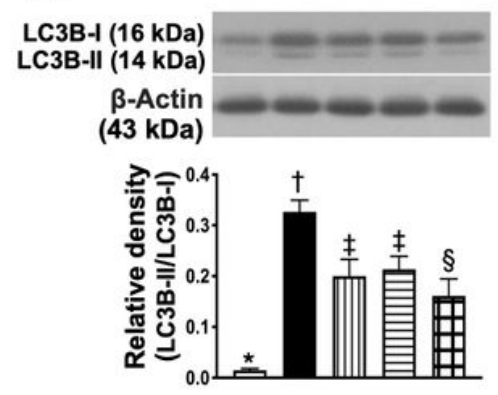

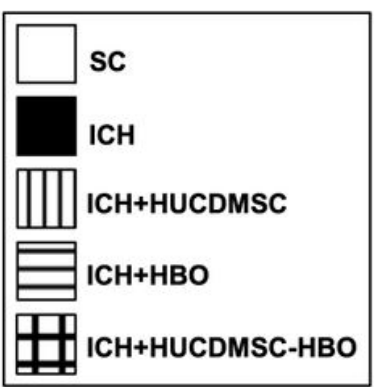

Figure 8 
Protein level of oxidative stress and autophagic biomarkers by day 28 after $\mathrm{ICH}$ induction A) Protein expressions of NOX-1, * vs. other groups with different symbols $(t, \neq, \S), p<0.0001$. B) Protein expression of NOX-2, * vs. other groups with different symbols $(t, \neq, \S), p<0.0001$. C) The oxidized protein expression, * vs. other groups with different symbols $(\dagger, \ddagger, \S), p<0.0001$ (Note: the left and right lanes shown on the upper panel represent protein molecular weight marker and control oxidized molecular protein standard, respectively). $\mathrm{M} \cdot \mathrm{W}=$ molecular weight; $\mathrm{DNP}=1-3$ dinitrophenylhydrazone. $\mathrm{D})$ Protein expressions of cleaved capspase $3(c-3)$, * vs. other groups with different symbols $(t, \neq, \S, \mathbf{9}), p<0.0001$. E) Protein expression of cleaved (c)-PARP, * vs. other groups with different symbols $(\dagger, \ddagger, \S), p<0.001$. F) Protein expression of ratio of LC3B-II to LC3B-l, * vs. other groups with different symbols $(t, \neq, \S), p<0.0001$. All statistical analyses were performed by one-way ANOVA, followed by Bonferroni multiple comparison post hoc test ( $n=6$ for each group). Symbols $(*,+, \neq, \S, 9)$ indicate significance (at 0.05 level). SC = shamoperated control; $\mathrm{ICH}=$ intracerebral hemorrhage; $\mathrm{HUCDMSC}=$ human umbilical cord-derived mesenchymal stem cell; $\mathrm{HBO}=$ hyperbaric oxygen . 


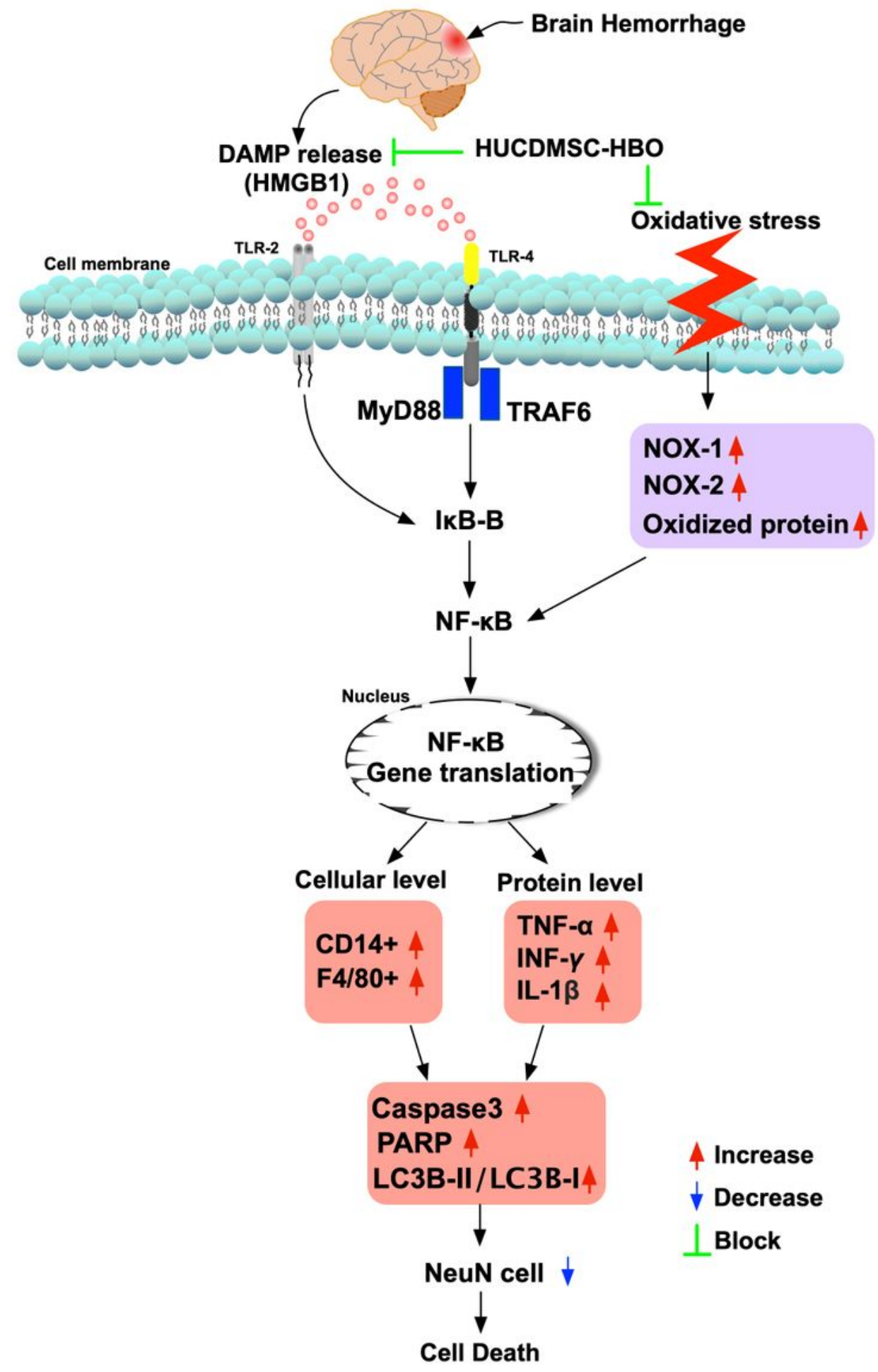

Figure 9

The proposed mechanism for how the intracerebral hemorrhage induced inflammatory signaling and oxidative stress and the impact of HUCDMSC + HBO therapy on improving the outcomes after IHC in rodent TLR = toll-like receptor; DAMP = damage-associated molecular patterns; HMGB1 = protein expression of high mobility group box $1 ;$ TRAF $6=\mathrm{TNF}$ receptor associated factor 6 . 